\author{
Marquette University \\ e-Publications@Marquette
}

Civil and Environmental Engineering Faculty

Research and Publications

Civil, Construction, and Environmental Engineering, Department of

\title{
$5-2011$
}

\section{Damping and Mass Sensitivity of Laterally Vibrating Resonant Microcantilevers in Viscous Liquid Media}

\author{
Russell Cox \\ Marquette University \\ Jinjin Zhang \\ Marquette University \\ Fabien Josse \\ Marquette University, fabien.josse@marquette.edu \\ Stephen M. Heinrich \\ Marquette University, stephen.heinrich@marquette.edu \\ Isabelle Dufour \\ Université de Bordeaux
}

See next page for additional authors

Follow this and additional works at: https://epublications.marquette.edu/civengin_fac

Part of the Civil and Environmental Engineering Commons

\section{Recommended Citation}

Cox, Russell; Zhang, Jinjin; Josse, Fabien; Heinrich, Stephen M.; Dufour, Isabelle; Beardslee, Luke A.; and Brand, Oliver, "Damping and Mass Sensitivity of Laterally Vibrating Resonant Microcantilevers in Viscous Liquid Media" (2011). Civil and Environmental Engineering Faculty Research and Publications. 53.

https://epublications.marquette.edu/civengin_fac/53 


\section{Authors}

Russell Cox, Jinjin Zhang, Fabien Josse, Stephen M. Heinrich, Isabelle Dufour, Luke A. Beardslee, and Oliver Brand 


\title{
Damping and mass sensitivity of laterally vibrating resonant microcantilevers in viscous liquid media
}

\author{
Russell Cox \\ Department Electrical and Computer Engineering, Marquette \\ University \\ Milwaukee, Wisconsin \\ Jinjin Zhang \\ Department Electrical and Computer Engineering, Marquette \\ University \\ Milwaukee, Wisconsin \\ Fabien Josse \\ Department Electrical and Computer Engineering, Marquette \\ University \\ Milwaukee, Wisconsin \\ Stephen M. Heinrich \\ Civil and Environmental Engineering, Marquette University \\ Milwaukee, Wisconsin \\ Isabelle Dufour \\ Université de Bordeaux CNRS, IMS Laboratory \\ Talence, France
}

[2011 Joint Conference of the IEEE International Frequency control and the European Frequency and the Time Forum, (May2011): pg. 1-6. DOI. This article is (C) Institute of Electrical and Electronics Engineers (IEEE) and permission has been granted for this version to appear in e-Publications@Marquette. Institute of Electrical and Electronics Engineers (IEEE) does not grant permission for this article to be further copied/distributed or hosted elsewhere without the express permission from Institute of Electrical and Electronics Engineers (IEEE).] 
NOT THE PUBLISHED VERSION; this is the author's final, peer-reviewed manuscript. The published version may be accessed by following the link in the citation at the bottom of the page.

\title{
Luke A. Beardslee
}

School of Electrical and Computer, Engineering, Georgia Institute of Technology

Atlanta, Georgia

\section{Oliver Brand}

School of Electrical and Computer, Engineering, Georgia Institute of Technology

Atlanta, Georgia

\begin{abstract}
The effect of liquid viscosity and density on the characteristics of laterally excited microcantilevers is investigated and compared to transversely excited microcantilevers. When immersed into a viscous liquid medium such as water from air, the resonant frequency of laterally (in-plane) vibrating microcantilevers is shown to decrease by only $5-10 \%$ as compared to $\sim 50 \%$ reduction for transversely (out-of-plane) vibrating microcantilevers. Furthermore, as the viscosity of the medium increases the resonant frequency of a laterally vibrating beam is shown to decrease at a slower rate than that of a transversely vibrating beam. The decreased viscous damping also leads to increases in the quality factor of the system by a factor of 4-5 compared to beams vibrating transversely. The mass sensitivities of laterally vibrating beams are also theoretically predicted to be roughly two orders of magnitude larger in water for some cantilever geometries. The increase in the quality factor and mass sensitivity indicate that operating in the in-plane flexural mode (lateral vibration) will decrease the limit of detection compared to operating in the more common out-of-plane flexural mode (transverse vibration). These improvements in device characteristics indicate that microcantilevers excited laterally are more suited for operating in media of high viscosities.
\end{abstract}

\section{SECTION I}

\section{INTRODUCTION}

Microcantilevers are microelectromechanical devices that can be used as highly sensitive chemical sensing platforms. When coated with a chemically selective layer, shifts in the resonant frequency of these beams can be related to changes in the ambient concentration of specific analytes in the medium of operation. A change in mass in the range of picograms has been detected using these devices, with

[2011 Joint Conference of the IEEE International Frequency control and the European Frequency and the Time Forum, (May2011): pg. 1-6. DOI. This article is (C) Institute of Electrical and Electronics Engineers (IEEE) and permission has been granted for this version to appear in e-Publications@Marquette. Institute of Electrical and Electronics Engineers (IEEE) does not grant permission for this article to be further copied/distributed or hosted elsewhere without the express permission from Institute of Electrical and Electronics Engineers (IEEE).] 
projected detection limits on the order of femtograms or attograms [1],[2]. However, when these beams are operating in a viscous liquid medium as opposed to air, the increased fluid damping and displaced fluid mass cause the resonant frequency and the mass sensitivity of the device to decrease. The increased fluid damping also causes the quality factor to decrease drastically, which increases the frequency noise and the limit of detection.

It was previously proposed that exciting microcantilevers in the in-plane flexural mode (instead of the more common out-of-plane flexural mode) would decrease the amount of viscous damping and displaced fluid mass, making the device a more appropriate platform for liquid phase sensing applications []ㅡ. In a previous work, a semianalytical expression for the hydrodynamic forces acting on laterally vibrating beams was derived to analyze these in-plane flexural mode cantilevers [ $\underline{3}]$. The expression predicts values for the hydrodynamic forces that compare well with other results presented in the literature [4] .

In the present study, this semi-analytical expression for the hydrodynamic forces will be used to predict the characteristics of laterally vibrating microcantilevers operating in media having higher viscosities than that of water (e.g., up to $20 \%$ aqueous glycerol solution). The characteristics of interest are the resonant frequency, the quality factor, and the mass sensitivity of the device. The predicted values for the resonant frequency and quality factor will be compared to experimentally obtained values, and the trends in these characteristics investigated. The predicted mass sensitivity for these devices will also be discussed, both as a function of the beam's geometry and as a function of the medium of operation. Finally, as the expressions for the characteristics of microcantilevers vibrating transversely are well-known, analytical expressions for the ratio of the characteristics of laterally and transversely vibrating beams of similar geometries will be determined. Such expressions will help to indicate the geometries and characteristics of interest for operation in the inplane flexural mode.

[2011 Joint Conference of the IEEE International Frequency control and the European Frequency and the Time Forum, (May2011): pg. 1-6. DOI. This article is (C) Institute of Electrical and Electronics Engineers (IEEE) and permission has been granted for this version to appear in e-Publications@Marquette. Institute of Electrical and Electronics Engineers (IEEE) does not grant permission for this article to be further copied/distributed or hosted elsewhere without the express permission from Institute of Electrical and Electronics Engineers (IEEE).] 
NOT THE PUBLISHED VERSION; this is the author's final, peer-reviewed manuscript. The published version may be accessed by following the link in the citation at the bottom of the page.

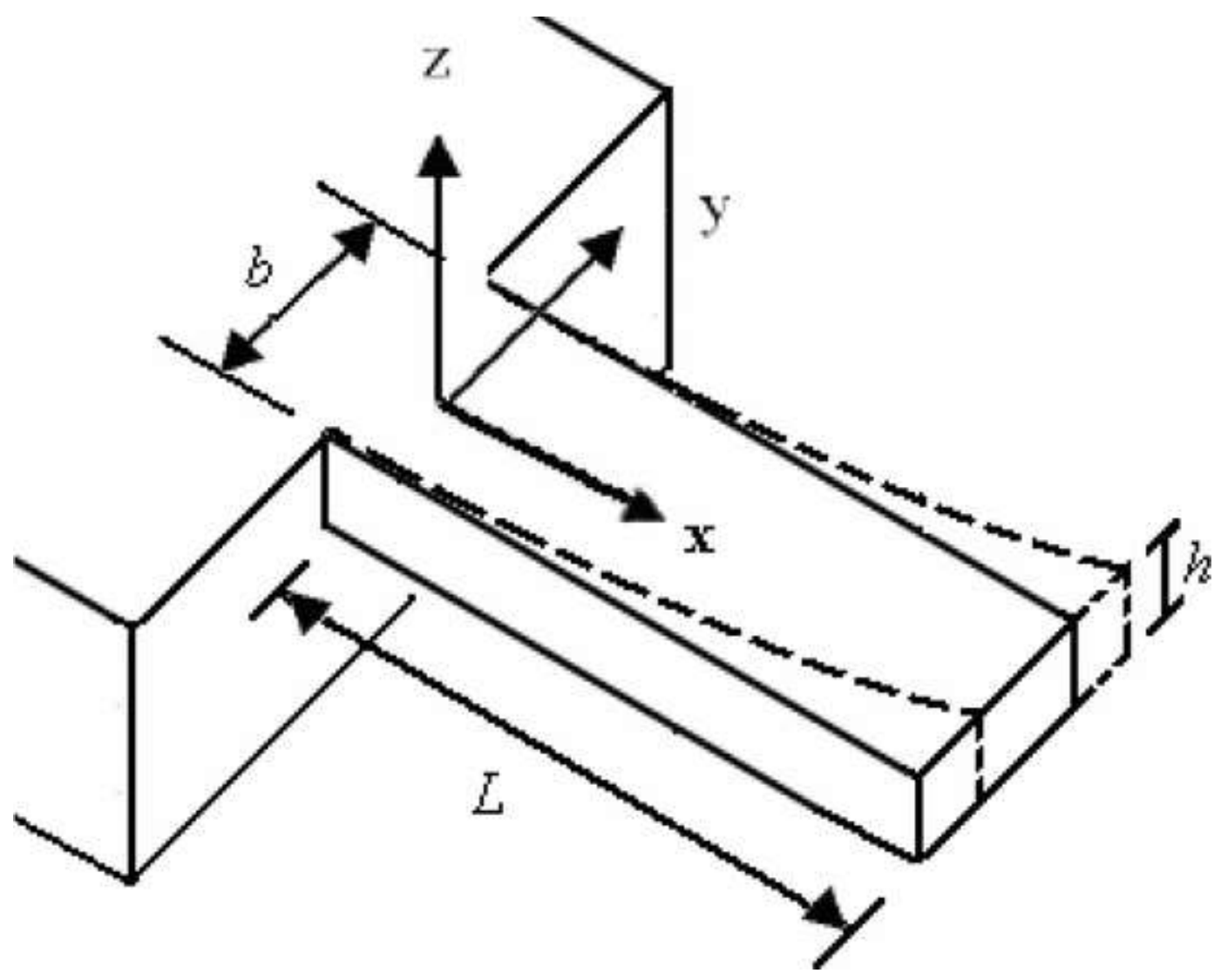

Figure 1: Geometry of a microcantilever of length $L$, width $b$, and thickness $h$ deflecting laterally

\section{SECTION II}

\section{THEORETICAL ANALYSIS}

\section{A. Resonant Frequency}

The geometry of a laterally excited microcantilever is shown in Fig. 1. The resonant frequency of a laterally vibrating beam in a viscous liquid medium was previously shown to be [ $\underline{3}]$

$$
f_{\text {res,lat }, i}=\frac{\alpha_{i}^{2}}{2 \pi} \sqrt{\frac{k_{\text {lat }}}{M_{\text {lat }}}}
$$

where $a_{i}$, is a constant dependent on the mode number $\left(\mathrm{a}_{1}=: 1.875\right.$ for the fundamental in-plane flexural mode) and the effective spring constant, $k_{\text {lat }}$, and effective mass, $M_{\text {lat }}$, are given by [2011 Joint Conference of the IEEE International Frequency control and the European Frequency and the Time Forum, (May2011): pg. 1-6. DOI. This article is (C) Institute of Electrical and Electronics Engineers (IEEE) and permission has been granted for this version to appear in e-Publications@Marquette. Institute of Electrical and Electronics Engineers (IEEE) does not grant permission for this article to be further copied/distributed or hosted elsewhere without the express permission from Institute of Electrical and Electronics Engineers (IEEE).] 
NOT THE PUBLISHED VERSION; this is the author's final, peer-reviewed manuscript. The published version may be accessed by following the link in the citation at the bottom of the page.

$$
\begin{gathered}
k_{\text {lat }}=\frac{E b^{3} h}{12 L^{3}} \\
M_{\text {lat }}=\left(\rho_{B} b h L+L g_{2, \text { lat }}\right) \\
+L \frac{\left(\left(g_{1, \text { lat }} / \omega_{\text {res,lat }}\right)+\left(\frac{\omega_{\text {lat }}}{2}\right) \frac{d}{d \omega}\left(g_{1, \text { lat }} / \omega\right)\right)}{\left(\rho_{B} b h+g_{2, \text { lat }}+\left(\frac{\omega_{\text {lat }}}{2}\right) \frac{d}{d \omega}\left(g_{2, \text { lat }}\right)\right)}\left(g_{1, \text { lat }} / \omega_{\text {lat }}\right)
\end{gathered}
$$

Here, $\rho B$ denotes the beam density and the

variables $g_{1, l a t}$ and $g_{2, l a t}$ are related to the amount of viscous damping and the amount of displaced fluid mass, respectively, and are given by

$$
\begin{aligned}
& g_{1, \text { lat }}=\frac{\pi}{4} \rho_{L} b^{2} \Gamma_{\text {lat }, l}(\operatorname{Re}, h / b) \omega_{\text {lat }}, \\
& g_{2, \text { lat }}=\frac{\pi}{4} \rho_{L} b^{2} \Gamma_{\text {lat }, R}(\operatorname{Re}, h / b) .
\end{aligned}
$$

The terms $\Gamma_{l a t, R}$ and $\Gamma_{l a t, l}$ are the real and imaginary parts, respectively, of the hydrodynamic function, a normalized version of the hydrodynamic force defined in [3] that depends only on the aspect ratio $h / b$ and the modified Reynolds number ( $\mathrm{Re}$ ) of the medium. The modified Reynolds number is a measure of the ratio of inertial forces to the viscous forces acting on the beam, and is defined as [ㅁ]

$$
\operatorname{Re}=\frac{\rho_{L} \omega_{l a t} b^{2}}{4 \eta}
$$

Where $\rho_{L}$ and $\eta$ are the mass density and dynamic viscosity of the fluid, respectively. It is assumed that the medium of operation is infinitely large and incompressible. It is also assumed that the velocity is continuous at the fluid-beam interface and the deflections of the beam are small compared to the dimensions of the beam. The beam's length is also assumed to be much greater than its width and thickness. Since the microcantilevers investigated in this study are composite beams made up of several layers, the Young's modulus, $E$, used in the theoretical predictions will be the effective Young's modulus

$$
E_{\text {effective }}=12 \rho_{B}\left(\frac{2 \pi L^{2} f_{0}}{\alpha_{i}^{2} b}\right)^{2}
$$

Where $f_{0}$ is the resonant frequency in vacuum. In this work, we used the experimentally determined resonant frequency of the beam in air instead, which has approximately the same value as the resonant

[2011 Joint Conference of the IEEE International Frequency control and the European Frequency and the Time Forum, (May2011): pg. 1-6. DOI. This article is (C) Institute of Electrical and Electronics Engineers (IEEE) and permission has been granted for this version to appear in e-Publications@Marquette. Institute of Electrical and Electronics Engineers (IEEE) does not grant permission for this article to be further copied/distributed or hosted elsewhere without the express permission from Institute of Electrical and Electronics Engineers (IEEE).] 
frequency in a vacuum. An effective beam density, $\rho B$, of $2330 \mathrm{~kg} / \mathrm{m}^{3}$ will be assumed as the density of each layer is approximately that of silicon.

Immersed in a fluid, the resonant frequency of a laterally vibrating beam decreases from its value in vaccum,/o, [6] by

$$
\Delta f=f_{0}\left(1-\sqrt{\frac{\rho_{B} L b h}{M_{l a t}}}\right) .
$$

As the viscous damping or displaced fluid mass increases, the total effective mass will increase, decreasing the resonant frequency. Since the amount of viscous damping is smaller when operating in the in-plane flexural mode compared to the out-of-plane flexural mode, the resonant frequency is expected to decrease by a smaller amount. The resonant frequency of the fundamental flexural mode of a laterally vibrating beam in a viscous liquid medium, , can be compared to the resonant frequency, , of the same beam vibrating transversely. Dividing (1) by the expression for the transverse resonant frequency in []], the ratio of these two values is predicted to be

$$
\frac{f_{\text {res,lat }}}{f_{\text {res,trans }}}=\frac{b}{h} \sqrt{\frac{M_{\text {trans }}}{M_{\text {lat }}}}
$$

where $M_{\text {trans }}$ is

$$
\begin{gathered}
M_{\text {trans }}=\left(\rho_{B} b h L+L g_{2, \text { trans }}\right) \\
+L \frac{\left(\left(g_{1, \text { trans }} / \omega_{\text {res,trans }}\right)+\left(\frac{\omega_{\text {trans }}}{2}\right) \frac{d}{d \omega}\left(g_{1, \text { trans }} / \omega\right)\right)}{\left(\rho_{B} b h+g_{2, \text { trans }}+\left(\frac{\omega_{\text {lat }}}{2}\right) \frac{d}{d \omega}\left(g_{2, \text { trans }}\right)\right)}\left(g_{1, \text { trans }} / \omega_{\text {trans }}\right)
\end{gathered}
$$

And $g_{1, \text { trans }}$ and $g_{2, \text { trans }}$ are defined by []]. Note that using thinner beams (or beams with larger values for $b / h$ ) will result in larger differences in their resonant frequency when operating in the inplane flexural mode. Also note that as the dynamic viscosity and density of the medium increases, the ratio of effective masses increases.

[2011 Joint Conference of the IEEE International Frequency control and the European Frequency and the Time Forum, (May2011): pg. 1-6. DOI. This article is (C) Institute of Electrical and Electronics Engineers (IEEE) and permission has been granted for this version to appear in e-Publications@Marquette. Institute of Electrical and Electronics Engineers (IEEE) does not grant permission for this article to be further copied/distributed or hosted elsewhere without the express permission from Institute of Electrical and Electronics Engineers (IEEE).] 
NOT THE PUBLISHED VERSION; this is the author's final, peer-reviewed manuscript. The published version may be accessed by following the link in the citation at the bottom of the page.

\section{B. Quality Factor}

Microcantilevers operating in the liquid-phase suffer from a drastic decrease in their quality factors. This decrease in the quality factor increases the frequency noise (which is proportional to $f_{\text {res,lat }} / Q_{\text {lat, }}$ when operating in an oscillator configuration [8] ), thus increasing the limit of detection in biochemical sensing applications. The quality factor is defined as $2 \Pi$ times the ratio of the maximum energy stored in a resonating system to the amount of energy dissipated in one cycle [9]. However, when a resonating device is used in an oscillator configuration, the quality factor is commonly obtained using the 3-dB bandwidth $\Delta f_{3 d B}$ of the device and is given by

$$
Q_{\text {lat, } 3 d B}=f_{\text {res,lat }} / \Delta f_{3 d B} .
$$

When the damping is small, $(8)$ represents a good approximation to the exact definition [9]. The quality factor of a laterally vibrating beam was previously derived as [3]

$$
Q_{\text {lat }}=\left(2\left(1-\sqrt{\frac{g_{1, \text { lat }} / \omega_{\text {lat }}}{\rho_{B} b h+g_{2, \text { lat }}}}\right)\right)^{-1}
$$

and, when $\operatorname{Re}>>1$, the quality factor can be approximated as

$$
Q_{\text {lat,approx }}=2 \pi f_{\text {res,lat }} \frac{\rho_{B} b h+g_{2, \text { lat }}}{g_{1, \text { lat }}} .
$$

The quality factor of laterally vibrating beams is predicted to be higher than that of transversely vibrating beams due to the decreased damping. The ratio of the quality factor of a laterally vibrating beam to that of a transversely vibrating beam of similar geometry is approximately given by

$$
\frac{Q_{\text {lat,approx }}}{Q_{\text {trans,approx }}}=\frac{\rho_{B} b h+g_{2, \text { lat }}}{\rho_{B} b h+g_{2, \text { trans }}} \frac{\Gamma_{I, \text { trans }}}{\Gamma_{I, \text { lat }}} .
$$

Note that the first fraction on the right hand side of $(11)$ is smaller than one (when $b>h$ ), as transversely vibrating beams displace more fluid compared to laterally vibrating beams. The second fraction is the ratio of the amounts of viscous damping which is normally much greater than one for the cases considered.

[2011 Joint Conference of the IEEE International Frequency control and the European Frequency and the Time Forum, (May2011): pg. 1-6. DOI. This article is (C) Institute of Electrical and Electronics Engineers (IEEE) and permission has been granted for this version to appear in e-Publications@Marquette. Institute of Electrical and Electronics Engineers (IEEE) does not grant permission for this article to be further copied/distributed or hosted elsewhere without the express permission from Institute of Electrical and Electronics Engineers (IEEE).] 
NOT THE PUBLISHED VERSION; this is the author's final, peer-reviewed manuscript. The published version may be accessed by following the link in the citation at the bottom of the page.

\section{Mass sensitivity}

The sensitivity of the resonant frequency to changes in the beam mass, or the mass sensitivity, can be defined as []]

$$
S_{m}=\left|\frac{\Delta f}{\Delta m}\right|
$$

An analytical expression for the mass sensitivity can be obtained using the same procedures described in []]. The expression for the mass sensitivity of a laterally vibrating beam is then the same as the expression for the transversely vibrating beam []], but with different values for the hydrodynamic function, , and the moment of inertia . If the thickness of the sensing layer is considered small enough, then the mass sensitivity can be given as

$$
S_{m}=\lambda_{m, l a t} f_{\text {res,lat }}
$$

Where

$$
\lambda_{m, \text { lat }}=\frac{\left(g_{1, \text { lat }} / \omega_{\text {lat }}\right)\left(g_{1, \text { lat }} / \omega_{\text {lat }}+\left(\frac{\omega_{\text {lat }}}{2}\right) \frac{d}{d \omega}\left(g_{1, \text { lat }} / \omega\right)\right) L^{2}}{2 M_{\text {lat }}\left(\rho_{B} b h L+L g_{2, \text { lat }}+L\left(\frac{\omega_{\text {lat }}}{2}\right) \frac{d}{d \omega}\left(g_{2, \text { lat }}\right)\right)^{2}}-\frac{1}{2 M_{\text {lat }}}
$$

The second term in (13a) dominates the first term when Re> $>1$. The mass sensitivity, $S_{m}$, can then be approximated as

$$
S_{\text {m,approx }}=\left|\frac{\alpha_{i}^{2}}{2 \pi} \frac{\sqrt{E b^{3} h L^{-3}}}{4 \sqrt{3}\left(M_{\text {lat }}\right)^{3 / 2}}\right| .
$$

When the beam is operating in air or low viscosity media, the effective mass can be approximated as the beam mass, $M_{\text {lat }} \cong \rho_{B} b h L$. In this case, the mass sensitivity has a geometric dependence of $h^{-1} L^{-3}$. As the decrease in the resonant frequency from air to water is small for laterally vibrating beams, this geometric dependency mostly holds when operating in a viscous liquid medium. Since the chemical sensitivity is proportional to the mass sensitivity multiplied by the volume of the sensing layer (e.g., $L b h_{2}$, where $h_{2}$ is the thickness of the sensing layer) the chemical sensitivity is predicted to be proportional to $\left(h_{2} / h\right) *\left(b / L^{2}\right)$. As the resonant frequency is known to be proportional to $b / L^{2}$ (e.g., []ㅡ), beams with higher resonant frequencies are then predicted to have higher chemical sensitivities. [2011 Joint Conference of the IEEE International Frequency control and the European Frequency and the Time Forum, (May2011): pg. 1-6. DOI. This article is (C) Institute of Electrical and Electronics Engineers (IEEE) and permission has been granted for this version to appear in e-Publications@Marquette. Institute of Electrical and Electronics Engineers (IEEE) does not grant permission for this article to be further copied/distributed or hosted elsewhere without the express permission from Institute of Electrical and Electronics Engineers (IEEE).] 
The ratio of the mass sensitivity of a laterally vibrating beam and that of the same beam vibrating transversely is also dependent on the beams' geometry. Using (14), this ratio can be approximated as

$$
\frac{S_{m, \text { lat }, \text { approx }}}{S_{m, \text { trans,approx }}}=\frac{f_{\text {res,lat }}}{f_{\text {res, }, \text { trans }}} \frac{M_{\text {trans }}}{M_{\text {lat }}}=\frac{b}{h}\left(\frac{M_{\text {trans }}}{M_{\text {lat }}}\right)^{3 / 2} .
$$

The effective mass of transversely vibrating beams is larger than that of laterally vibrating beams. Thus, the increase in the mass sensitivity when using lateral excitation compared with transverse excitation will be equal to or greater than $b / h$. Note again that thinner beams will benefit more when operating in the in-plane flexural mode than thicker beams. Also note that this ratio will be larger than the ratio of the resonant frequencies by a factor of $M_{\text {trans }} / M_{\text {lat }}$.

\section{SECTION III}

\section{RESULTS AND DISCUSSION}

Experimental results for the resonant frequency and the quality factor of laterally vibrating beams both in water and aqueous mixtures of glycerol were obtained. The set-up used to extract the experimental data is described in [10]. The microcantilevers were laterally excited using thermal excitation. The beam's deflection as a function of excitation frequency was obtained using a Wheatstone bridge fabricated on the beam with the sensing resistors oriented so that the bridge voltage could be related to the lateral deflection of the beam. Solutions of varying aqueous percent glycerol (from pure water to $20 \% \mathrm{w} / \mathrm{w}$ ) were placed in a flow cell containing the microcantilevers so that the beams were completely immersed in the solution. The spectrum of each device was then obtained and the resonant frequency and quality factor of the first in-plane flexural mode were extracted. Several microcantilevers were tested and sample results will be discussed for selected geometries. The silicon-based microcantilevers used in this work have a nominal silicon thickness of either 4.8 or $9.0 \mu \mathrm{m}$ and are covered with a $1.9 \mu \mathrm{m}$ passivation layer on the top made up of alternating layers of silicon nitride and silicon oxide (for fabrication details, see [10]).

[2011 Joint Conference of the IEEE International Frequency control and the European Frequency and the Time Forum, (May2011): pg. 1-6. DOI. This article is (C) Institute of Electrical and Electronics Engineers (IEEE) and permission has been granted for this version to appear in e-Publications@Marquette. Institute of Electrical and Electronics Engineers (IEEE) does not grant permission for this article to be further copied/distributed or hosted elsewhere without the express permission from Institute of Electrical and Electronics Engineers (IEEE).] 
The decrease in the resonant frequency of a $200 \times 60 \times 6.7 \mu \mathrm{m}^{3}$ laterally vibrating beam due to the increase in the viscous damping and displaced fluid mass as a function of the percent aqueous glycerol is shown in Fig. 2. The theoretical results calculated using (1) show similar trends; however, the decrease in the experimentally determined resonant frequency is smaller than the decrease in the theoretically predicted resonant frequency. The predicted resonant frequency in water is slightly different from the experimentally determined value ( $1390.7 \mathrm{kHz}$ vs. $1381.3 \mathrm{kHz}$, respectively). The predicted decrease in the resonant frequency also has better agreement with the experimental results for longer beams, i.e. $1000 \times 90 \times 10.9 \mu \mathrm{m}^{3}$ beam. This suggests that the difference could be due to effects that are not accounted for when the assumption of a long beam is made. This assumption neglects the effects of the shear and rotational inertia of the beam, which cause the resonant frequency to be lower. These effects tend to be larger for shorter beams.

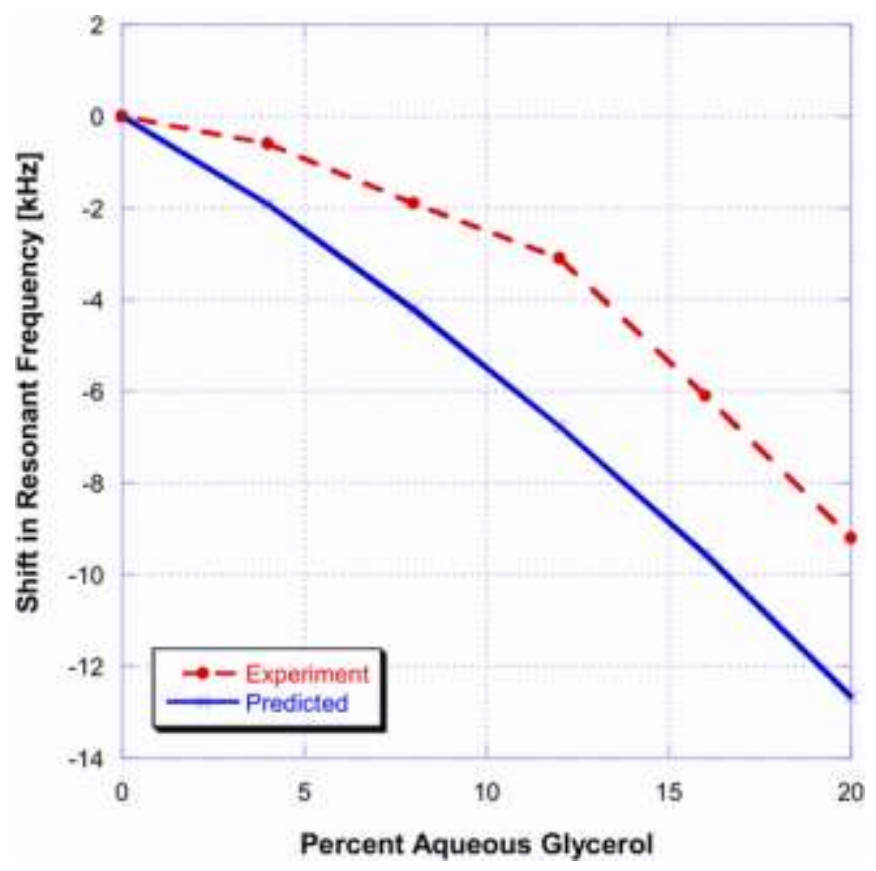

Figure 2: Predicted and experimentally determined shift in the resonant frequency of a $200 \times 60 \times 6.7 \mu \mathrm{m}$ laterally vibrating microcantilever from water to a solution of aqueous glycerol.

[2011 Joint Conference of the IEEE International Frequency control and the European Frequency and the Time Forum, (May2011): pg. 1-6. DOI. This article is (C) Institute of Electrical and Electronics Engineers (IEEE) and permission has been granted for this version to appear in e-Publications@Marquette. Institute of Electrical and Electronics Engineers (IEEE) does not grant permission for this article to be further copied/distributed or hosted elsewhere without the express permission from Institute of Electrical and Electronics Engineers (IEEE).] 
NOT THE PUBLISHED VERSION; this is the author's final, peer-reviewed manuscript. The published version may be accessed by following the link in the citation at the bottom of the page.

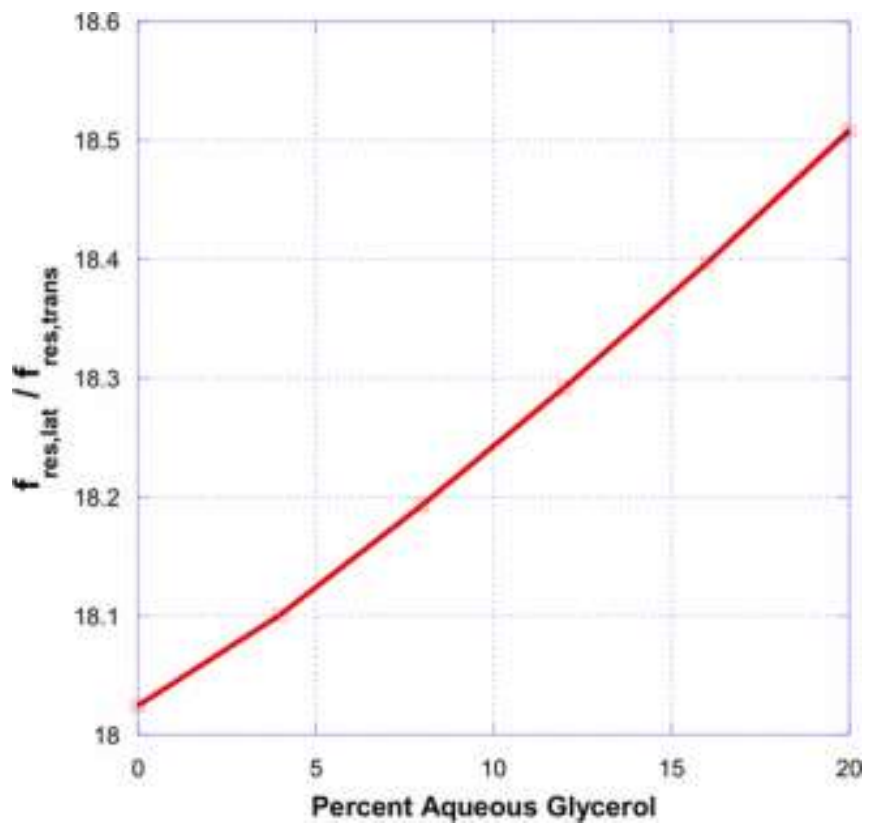

Figure 3: The predicted ratios of the first lateral and transverse resonant frequencies as a function of the medium's percent aqueous glycerol for a $200 \times 60 \times 6.7 \mu \mathrm{m}$ beam

Due to the increased stiffness of operating in the in-plane flexural mode, the resonant frequencies of laterally vibrating beams will be higher than those of transversely vibrating beams of similar geometry. When the effective mass can be approximated by the beam mass (such as when operating in vacuum or air), the predicted ratio of resonant frequencies of laterally and transversely vibrating microcantilevers is approximately equal to the ratio of their width and thickness. When the beam is operating in a more viscous liquid medium, this ratio of frequencies increases. The predicted ratio of the resonant frequencies of a laterally and transversely vibrating $200 \times 60 \times$ $6.7 \mu \mathrm{m}^{3}$ microcantilever is given in Fig. 3 as a function of the percent aqueous glycerol in the operational medium. This ratio is larger than the width to thickness ratio of 8.95 due to the differences in the effective mass. As noted before, the resonant frequency of a laterally vibrating beam decreases with increasing percent aqueous glycerol. This means that the increase in the ratio is due to the transverse resonant frequency dropping more drastically than its lateral counterpart as the viscosity is increased. Because a high resonant frequency is desired for sensing applications, it is thus more

[2011 Joint Conference of the IEEE International Frequency control and the European Frequency and the Time Forum, (May2011): pg. 1-6. DOI. This article is (C) Institute of Electrical and Electronics Engineers (IEEE) and permission has been granted for this version to appear in e-Publications@Marquette. Institute of Electrical and Electronics Engineers (IEEE) does not grant permission for this article to be further copied/distributed or hosted elsewhere without the express permission from Institute of Electrical and Electronics Engineers (IEEE).] 
NOT THE PUBLISHED VERSION; this is the author's final, peer-reviewed manuscript. The published version may be accessed by following the link in the citation at the bottom of the page.

advantageous to operate in the in-plane flexural mode when operating in high-viscosity media.

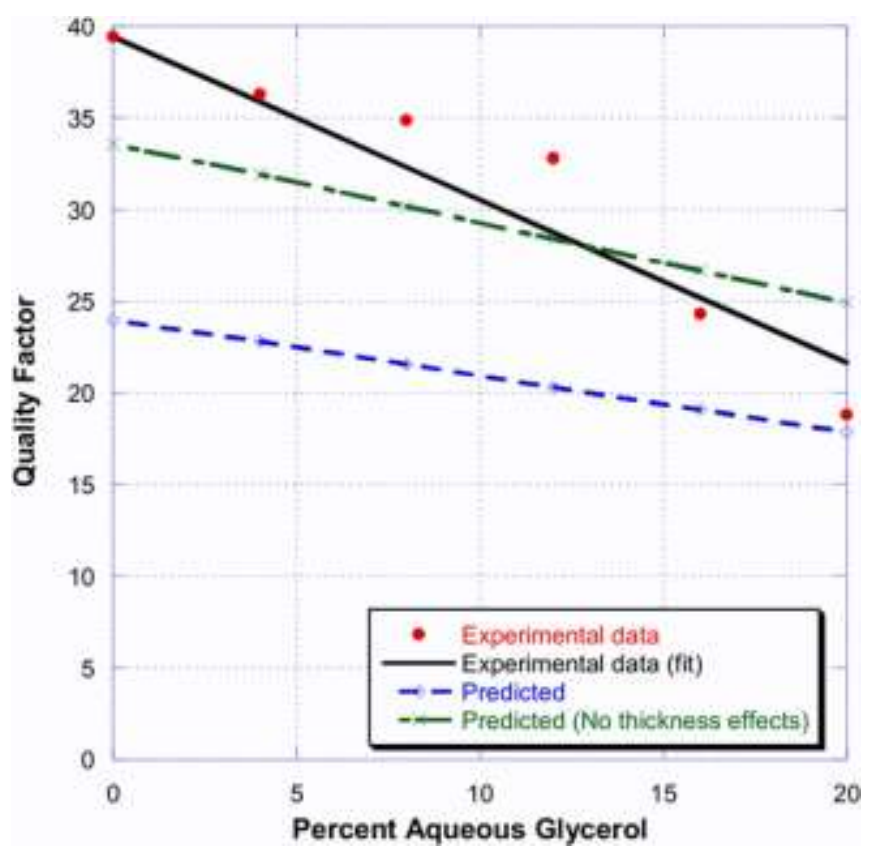

Figure 4: Predicted and experimentally determined quality factors of a $200 \times 60 \times$ $6.7 \mu \mathrm{m}$ laterally vibrating microcantilever as a function of percent aqueous glycerol.

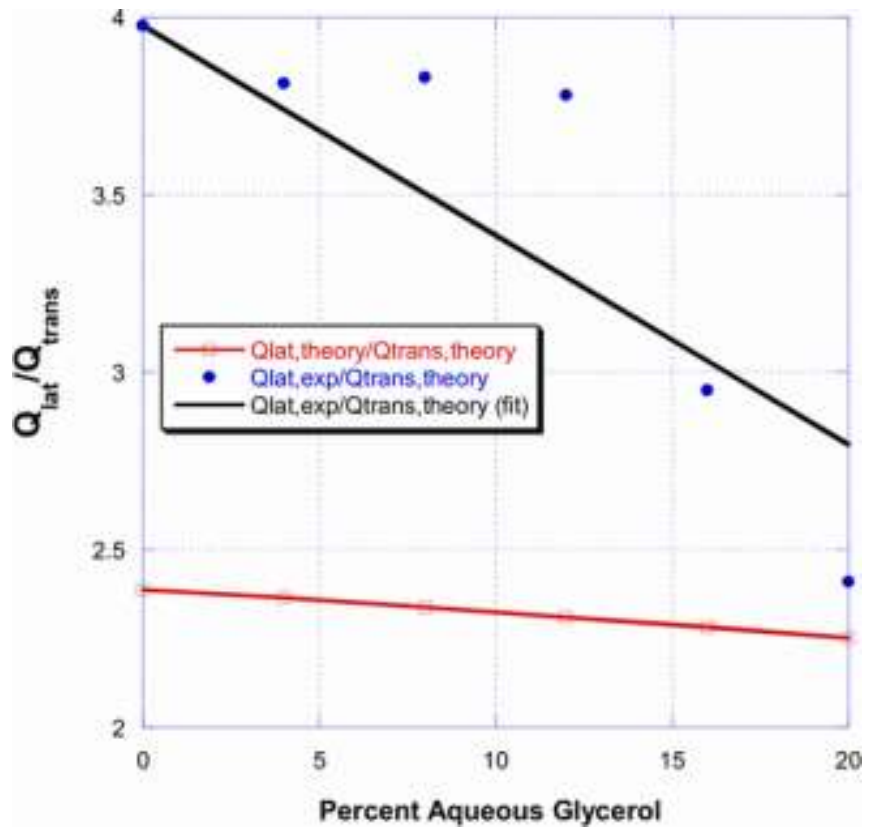

Figure 5: The ratios of the quality factors of laterally (both predicted and experimentally determined) and transversely vibrating beams $200 \times 60 \times 6.7 \mu \mathrm{m}$ as a function of percent aqueous glycerol (medium of operation).

[2011 Joint Conference of the IEEE International Frequency control and the European Frequency and the Time Forum, (May2011): pg. 1-6. DOI. This article is (C) Institute of Electrical and Electronics Engineers (IEEE) and permission has been granted for this version to appear in e-Publications@Marquette. Institute of Electrical and Electronics Engineers (IEEE) does not grant permission for this article to be further copied/distributed or hosted elsewhere without the express permission from Institute of Electrical and Electronics Engineers (IEEE).] 
NOT THE PUBLISHED VERSION; this is the author's final, peer-reviewed manuscript. The published version may be accessed by following the link in the citation at the bottom of the page.

The quality factor as a function of percent aqueous glycerol for the same beam $200 \times 60 \times 6.7 \mu \mathrm{m}^{3}$ is given in Fig. 4. The experimentally determined quality factors are larger than the commonly obtained values for transversely vibrating beams, which are usually on the order of 10 [11]. While the experimental results show similar trends as the theoretically predicted results, the experimentally determined quality factors are larger than the theoretically predicted quality factors. Calculated values for the quality factor using a simpler expression given by [12] are also shown in Fig. 4. However, this expression neglects the pressure effects of the fluid on the smaller faces (of dimension $h$ ), i.e., it is based on assuming only a Stokes-type fluid resistance on the top and bottom faces of the beam. The theoretically predicted quality factors would be smaller if the shear deformation and support compliance effects were taken into account. Thus, these effects are not the source of the discrepancy. Another assumption made when the hydrodynamic forces were estimated in [3] was that the beam was infinitely long. This assumption was made to neglect the edge effects near the end of the beam in the lengthwise direction, and might not be appropriate for the given geometry. It is noted that the quality factors of longer beams (e.g. $1000 \times 90 \times 10.9 \mu \mathrm{m}^{3}$ ) more closely match the characteristics predicted by theory.

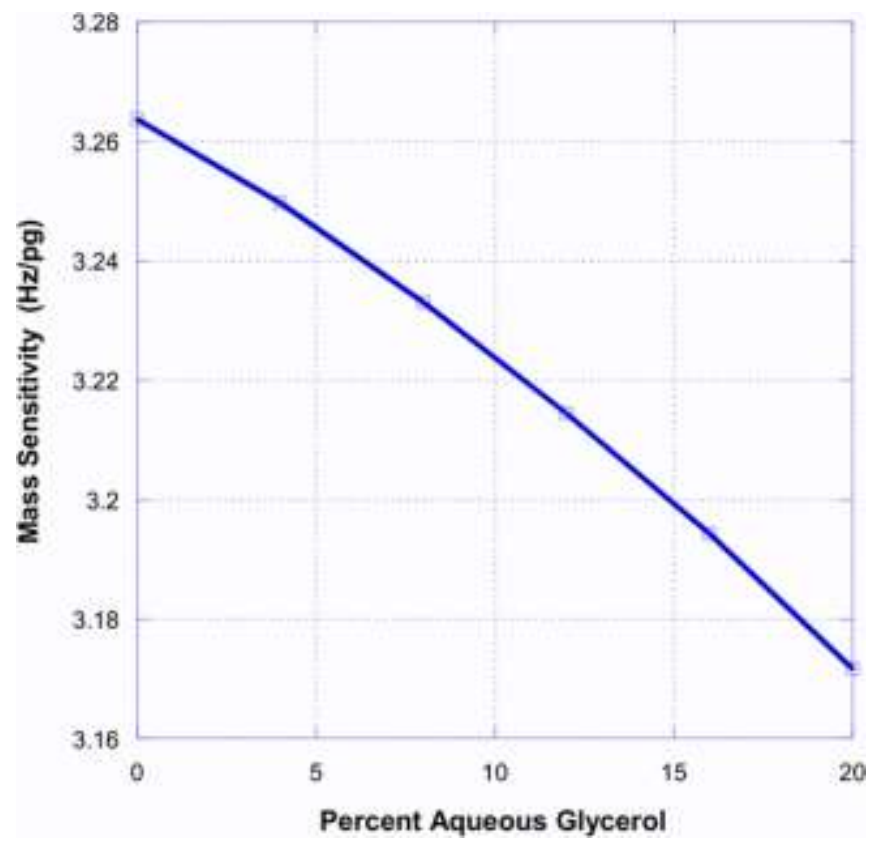

[2011 Joint Conference of the IEEE International Frequency control and the European Frequency and the Time Forum, (May2011): pg. 1-6. DOI. This article is (C) Institute of Electrical and Electronics Engineers (IEEE) and permission has been granted for this version to appear in e-Publications@Marquette. Institute of Electrical and Electronics Engineers (IEEE) does not grant permission for this article to be further copied/distributed or hosted elsewhere without the express permission from Institute of Electrical and Electronics Engineers (IEEE).] 
NOT THE PUBLISHED VERSION; this is the author's final, peer-reviewed manuscript. The published version may be accessed by following the link in the citation at the bottom of the page.

Figure 6: Predicted mass sensitivity of a $200 \times 60 \times 6.7 \mu m$ laterally vibrating microcantilever as a function of percent aqueous glycerol.

Regardless of the length used, the quality factor tends to be higher when operating in the in-plane flexural mode compared to the out-of-plane flexural mode. It is predicted, when operating in air, that the quality factor of a $200 \times 60 \times 6.7 \mu \mathrm{m}$ beam is a factor of 6.16 times larger for the in-plane flexural mode than for the out-of-plane flexural mode. This increase, unlike the resonant frequency, is expected to be smaller when operating in a viscous liquid medium. Fig. 5 shows the predicted ratio of the quality factor of a $200 \times 60 \times 6.7 \mu \mathrm{m}^{3}$ laterally vibrating silicon microcantilever and that of a transversely vibrating microcantilever of the same geometry as a function of percent aqueous glycerol. As the experimental results show higher quality factor values than the predicted theoretical results, this improvement is expected to be higher when using the experimental results for comparison. The improvement in the quality factor is also a function of the beam's geometry. For example, longer and narrower beams will have a smaller increase in quality factor when compared to shorter and wider beams. This is because shorter and wider beams have higher resonant frequencies, Reynolds numbers, and thus larger damping ratios.

The mass sensitivity was not experimentally determined in this study. However, the mass sensitivity can still be estimated for the microcantilever as a function of percent aqueous $200 \times 60 \times$ $6.7 \mu \mathrm{m}^{3}$ glycerol, and is given in Fig. 6. While the predicted mass sensitivity is expected to decrease as the percent aqueous glycerol increases, the rate of decrease is much smaller than that of similar beams vibrating transversely. For example, a $200 \times 60 \times 6.7 \mu \mathrm{m}^{3}$ laterally vibrating microcantilever in water is predicted to have a $3.26 \mathrm{~Hz} / \mathrm{pg}$ mass sensitivity compared to a $0.0443 \mathrm{~Hz} / \mathrm{pg}$ mass sensitivity of the same beam vibrating transversely. When the viscosity of the operational environment is increased to 1.734 cP (20\% aqueous glycerol), the mass sensitivity of the transversely vibrating beam decreases by $10.4 \%$ to $0.0397 \mathrm{~Hz} / \mathrm{pg}$, while the same beam laterally vibrating only decreases by $2.76 \%$ to $3.17 \mathrm{~Hz} / \mathrm{pg}$. This indicates a further improvement in more viscous fluid. The ratio of the mass sensitivity as a function of percent aqueous glycerol is given in Fig. 7 .

[2011 Joint Conference of the IEEE International Frequency control and the European Frequency and the Time Forum, (May2011): pg. 1-6. DOI. This article is (C) Institute of Electrical and Electronics Engineers (IEEE) and permission has been granted for this version to appear in e-Publications@Marquette. Institute of Electrical and Electronics Engineers (IEEE) does not grant permission for this article to be further copied/distributed or hosted elsewhere without the express permission from Institute of Electrical and Electronics Engineers (IEEE).] 
NOT THE PUBLISHED VERSION; this is the author's final, peer-reviewed manuscript. The published version may be accessed by following the link in the citation at the bottom of the page.

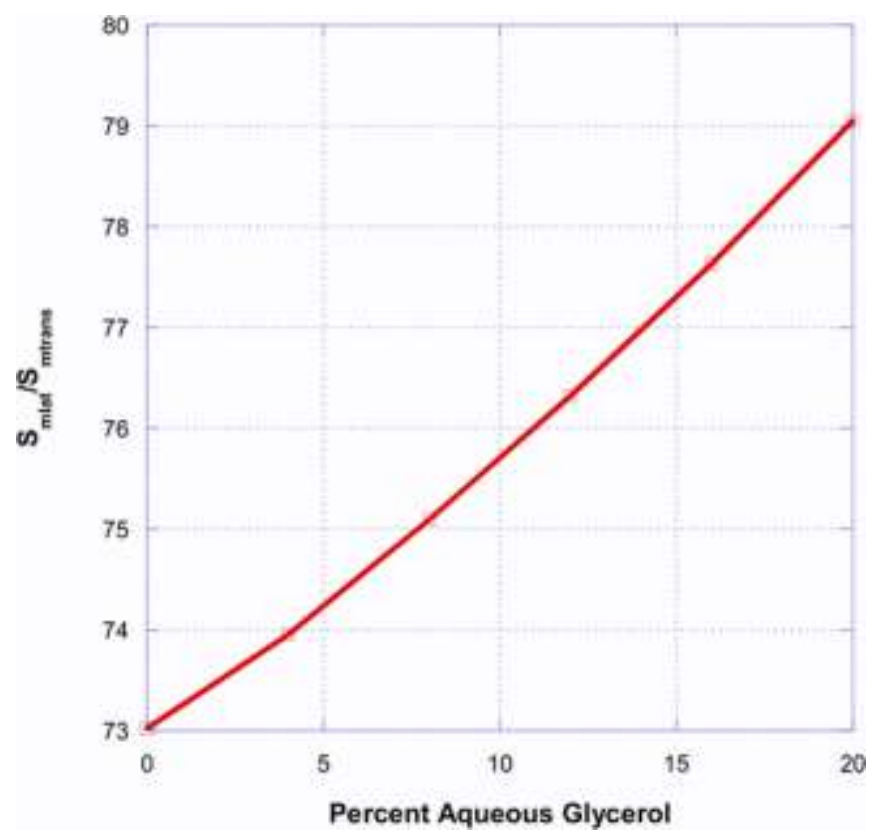

Figure 7: The ratio of the mass sensitivities of a laterally and a transversely vibrating beam for a $200 \times 60 \times 6.7 \mu \mathrm{m}$ microcantilever as a function of percent aqueous glycerol in the operational medium.

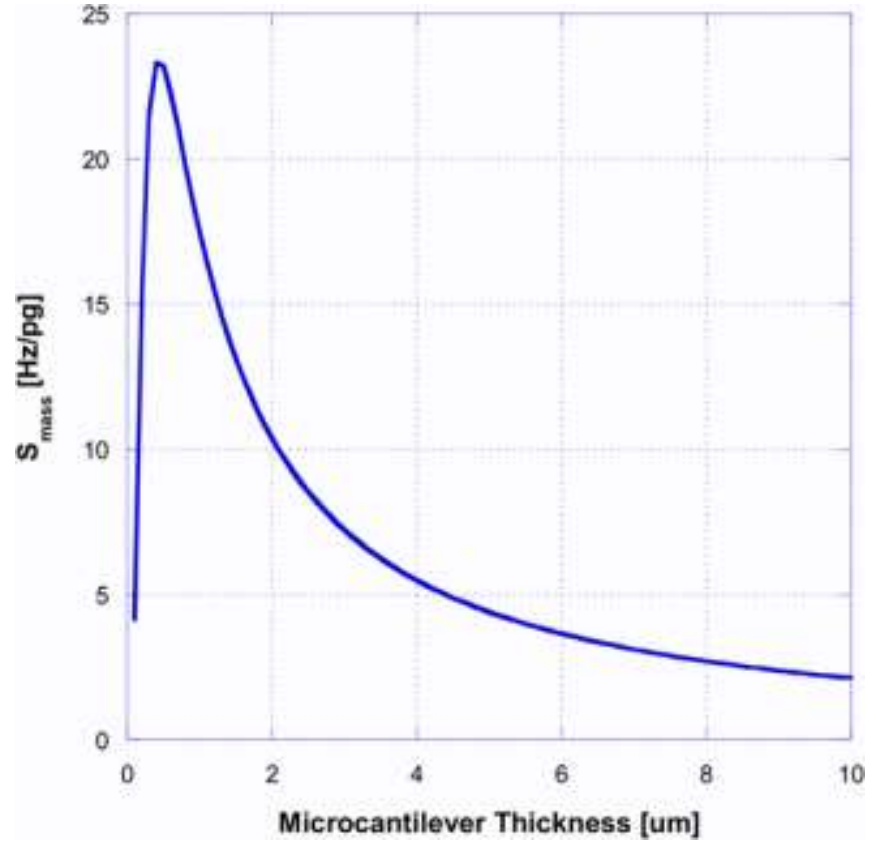

Figure 8. The mass sensitivity of a laterally vibrating $200 \times 60 \mu m$ microcantilever in water as a function of beam thickness.

[2011 Joint Conference of the IEEE International Frequency control and the European Frequency and the Time Forum, (May2011): pg. 1-6. DOI. This article is (C) Institute of Electrical and Electronics Engineers (IEEE) and permission has been granted for this version to appear in e-Publications@Marquette. Institute of Electrical and Electronics Engineers (IEEE) does not grant permission for this article to be further copied/distributed or hosted elsewhere without the express permission from Institute of Electrical and Electronics Engineers (IEEE).] 
The ratio of the chemical sensitivities, assuming the same geometry and sensing layer, is the same as the ratio of the mass sensitivities. If the thickness of the beam is decreased, this ratio would be even larger, as the mass sensitivity is also a function of the beam's geometry. Shorter and thinner beams are expected to have higher mass sensitivities. The predicted mass sensitivity of a $200 \times$ $60 \mu \mathrm{m}^{2}$ silicon microcantilever in water as a function of thickness is given in Fig. 8. Note that the mass sensitivity is predicted to initially increase with increasing thickness. This increase occurs because, for small thicknesses, the fluid mass dominates the beam mass. It's noted that the stiffness is linearly dependent on the thickness. When the beam mass dominates the fluid mass as the thickness increases, the mass sensitivity decreases. However, as discussed in [3] , the quality factor will also increase with increasing thickness. As the limit of detection is a function of both the chemical sensitivity (which depends on the mass sensitivity) and the quality factor, both must be considered when choosing the appropriate thickness of a microcantilever for a given application. However, increasing the width will increase the quality factor while not greatly affecting the mass sensitivity of laterally vibrating beams; and decreasing the length will increase both the quality factor and the mass sensitivity of laterally vibrating beams.

\section{SECTION IV}

\section{CONCLUSIONS}

It is shown that laterally vibrating microcantilevers can be excited and have relatively high (20 to 60 ) quality factors, even when operating in media with higher viscosity than water. The resonant frequency of laterally vibrating beams in a viscous liquid medium is also observed to experience relatively insignificant decreases (compared to its resonant frequency in air). When compared to transversely vibrating beams, the predicted characteristics (i.e. the resonant frequency, quality factor, and mass sensitivity) are shown to be higher. Considering the resonant frequency and mass sensitivity, this increase is larger for media with higher dynamic viscosities. These improvements result in an almost two orders of magnitude higher chemical sensitivity in viscous solutions. Besides for chemical sensing, [2011 Joint Conference of the IEEE International Frequency control and the European Frequency and the Time Forum, (May2011): pg. 1-6. DOI. This article is (C) Institute of Electrical and Electronics Engineers (IEEE) and permission has been granted for this version to appear in e-Publications@Marquette. Institute of Electrical and Electronics Engineers (IEEE) does not grant permission for this article to be further copied/distributed or hosted elsewhere without the express permission from Institute of Electrical and Electronics Engineers (IEEE).] 
this is ideal for biosensor applications where biofluids may have higher viscosities than water. It is also shown that shorter and wider beams will have higher quality factors and larger improvements in their mass sensitivity compared to transversely vibrating microcantilevers. This indicates that the limit of detection of microcantilever bio-chemical sensors operating in the in-plane flexural mode will be lower than that of microcantilevers operating in the out-of-plane flexural mode in liquid-phase sensing applications.

\section{ACKNOWLEDGMENTS}

This work has been funded in part by the National Science Foundation under award ECCS-0824017

\section{REFERENCES}

L. A. Pinnaduwage, V. Boiadjiev, J. E. Hawk, and T. Thundat, "Sensitive Detection of Plastic Explosives with Self-Assembled Monolayer-coated Microcantilevers," Applied Physics Letters, vol. 83, no. 7, p. 1471, 2003.

J. Davis, G. Abadal, O. Kuhn, O. Hansen, F. Grey, and A. Boisen, "Fabrication and Characterization of Nanoresonating Devices for Mass Detection," Journal of Vacuum Science \& Technology B, vol. 18, no. 2, pp. 612-616, 2000.

R. Cox, F. Josse, S. Heinrich, I. Dufour, O. Brand, "Resonant Microcantilevers Vibrating Laterally in Viscous Liquid Media," IEEE International Frequency Control Symposium (FCS), pp. 8590, 2010.

D. Brumley, M. Willcox, and, J. Sader, "Oscillation of Cylinders of Rectangular Cross section Immersed in Fluid," Physics of Fluids, vol. 22, no. 052001, 2010.

J. Sader, "Frequency Response of Cantilever Beams Immersed in Viscous Fluids with Applications to the Atomic Force Microscope," Journal of Applied Physics, vol. 84, no. 1, pp. 6476, July 1998.

[2011 Joint Conference of the IEEE International Frequency control and the European Frequency and the Time Forum, (May2011): pg. 1-6. DOI. This article is (C) Institute of Electrical and Electronics Engineers (IEEE) and permission has been granted for this version to appear in e-Publications@Marquette. Institute of Electrical and Electronics Engineers (IEEE) does not grant permission for this article to be further copied/distributed or hosted elsewhere without the express permission from Institute of Electrical and Electronics Engineers (IEEE).] 
I. Dufour, S. Heinrich, and F. Josse, "Theoretical Analysis of StrongAxis Bending Mode Vibrations for Resonant Microcantilever (Bio)Chemical Sensors in Gas or Liquid Phase," Journal of Microelectromechanical Systems, vol.16, iss.1, pp. 44-49, Feb. 2007.

R. Cox, F. Josse, M. J. Wenzel, S. Heinrich, and I. Dufour, "Generalized Model of Resonant Polymer-Coated Microcantilevers in Viscous Liquid Media," Analytical Chemistry, vol. 80, no. 15, pp. 57605767, 2008.

I. Dufour, F. Lochon, S. Heinrich, F. Josse, D. Rebière, "Effect of Coating Viscoelasticity on Quality Factor and Limit of Detection of Microcantilever Chemical Sensors ", IEEE Sensors Journal, vol. 7, 2007, pp. 230-236

B. Razavi, "A Study of Phase Noise in CMOS Oscillators," IEEE Journal of Solid-State Circuits, vol. 31, no. 3, pp. 331-343, 1996.

L. Beardslee, A. M. Addous, S. Heinrich, F. Josse, I. Dufour, and O. Brand, "Thermal Excitation and Piezoresistive Detection of Cantilever In-Plane Resonance Modes for Sensing Applications," Journal of Microelectromechanical Systems, vol. 19, no. 4, pp. 1015-1017, 2010.

J. Chon, P. Mulvaney, and J. Sader, "Experimental Validation of Theoretical Models for the Frequency Response of Atomic Force Microscope Cantilever Beams Immersed in Fluids," Journal of Applied Physics, vol. 87, no. 8, pp. 3978-3988, April 2000.

S. M. Heinrich, R. Maharjan, I. Dufour, F. Josse, L. Beardslee and O. Brand, "An Analytical Model of a Thermally Excited Microcantilever Vibrating Laterally in a Viscous Fluid," Proceedings IEEE Sensors 2010 Conference, Waikoloa, Hawaii, pp. 1399-1404., 2010

[2011 Joint Conference of the IEEE International Frequency control and the European Frequency and the Time Forum, (May2011): pg. 1-6. DOI. This article is (C) Institute of Electrical and Electronics Engineers (IEEE) and permission has been granted for this version to appear in e-Publications@Marquette. Institute of Electrical and Electronics Engineers (IEEE) does not grant permission for this article to be further copied/distributed or hosted elsewhere without the express permission from Institute of Electrical and Electronics Engineers (IEEE).] 